\title{
Erratum to: Risk factors for current and future unmet supportive care needs of people with pancreatic cancer. A longitudinal study
}

\author{
Vanessa L Beesley ${ }^{1}$ - Leesa F Wockner ${ }^{1}$ • Peter O'Rourke ${ }^{1}$ - Monika Janda ${ }^{2}$. \\ David Goldstein $^{3,4}$ • Helen Gooden ${ }^{5}$. Neil D Merrett ${ }^{6,7}$ - Dianne L O'Connell ${ }^{8}$. \\ Ingrid J Rowlands $^{9} \cdot$ David K Wyld ${ }^{10,11} \cdot$ Rachel E Neale ${ }^{1}$
}

Published online: 6 May 2016

(C) Springer-Verlag Berlin Heidelberg 2016

\section{Erratum to: Support Care Cancer}

10.1007/s00520-016-3212-4

Table 1 was printed incorrectly during the typesetting stage and has since been corrected. Please find below the corrected version of Table 1. Springer regrets the error.

The online version of the original article can be found at http://dx.doi.org/ $10.1007 / \mathrm{s} 00520-016-3212-4$.

Vanessa L Beesley

Vanessa.Beesley@qimrberghofer.edu.au

1 Population Health Department, QIMR Berghofer Medical Research Institute, Brisbane, Australia

2 School of Public Health and Social Work, Queensland University of Technology, Brisbane, Australia

3 Department of Medical Oncology, Prince of Wales Hospital, Sydney, Australia

4 University of New South Wales, Sydney, Australia

5 Cancer Nursing Research Unit, University of Sydney, Sydney, Australia
Discipline of Surgery, Western Sydney University, Sydney, Australia

7 SouthWestern Sydney Upper GI Surgical Unit, Bankstown Hospital, Sydney, Australia

8 Cancer Research Division, Cancer Council New South Wales, Sydney, Australia

9 School of Public Health, The University of Queensland, Brisbane, Australia

10 Department of Medical Oncology, Royal Brisbane and Women's Hospital, Brisbane, Australia

11 University of Queensland, Brisbane, Australia 
Table 1 Baseline demographic and clinical characteristics of participants and bivariable associations with any baseline moderate-to-high unmet needs

\begin{tabular}{|c|c|c|c|c|}
\hline & Responders $(n=116)$ & $\begin{array}{l}\text { Participants with } \\
\text { no-to-low unmet } \\
\text { needs }(n=35) \\
n(\%)\end{array}$ & $\begin{array}{l}\text { Participants with } \geq 1 \\
\text { moderate-to-high } \\
\text { unmet need }(n=81) \\
\mathrm{n}(\%)\end{array}$ & $p$-value ${ }^{\mathrm{a}}$ \\
\hline \multicolumn{5}{|l|}{ Age (years) } \\
\hline Mean (SD) & $67.0(9.5)$ & $68.0(8.3)$ & $66.6(10.0)$ & 0.500 \\
\hline \multicolumn{5}{|l|}{ Sex } \\
\hline Male & $70(60)$ & $22(31)$ & $48(69)$ & \multirow{2}{*}{0.716} \\
\hline Female & $46(40)$ & $13(28)$ & $33(72)$ & \\
\hline \multicolumn{5}{|l|}{ Marital Status } \\
\hline Married/partner & $93(80)$ & $28(30)$ & $65(70)$ & \multirow{2}{*}{0.976} \\
\hline Divorce/separated/widowed/never married & $23(20)$ & $7(30)$ & $16(70)$ & \\
\hline \multicolumn{5}{|l|}{ Education } \\
\hline High school or lower & $48(42)$ & $15(31)$ & $33(69)$ & \multirow[t]{2}{*}{0.738} \\
\hline College or higher & $67(58)$ & $19(28)$ & $48(72)$ & \\
\hline \multicolumn{5}{|l|}{ Place of residence } \\
\hline Major city & $54(47)$ & $15(28)$ & $39(72)$ & \multirow[t]{3}{*}{0.570} \\
\hline Inner regional & $52(45)$ & $18(35)$ & $34(65)$ & \\
\hline Outer regional & $10(9)$ & $2(20)$ & $8(80)$ & \\
\hline \multicolumn{5}{|l|}{ Months post-diagnosis at questionnaire 1} \\
\hline Mean (SD) & $2.9(1.3)$ & $3.1(1.5)$ & $2.8(1.3)$ & 0.344 \\
\hline \multicolumn{5}{|l|}{ Initial place of treatment } \\
\hline Public hospital & $43(37)$ & $12(28)$ & $31(72)$ & \multirow[t]{2}{*}{0.728} \\
\hline Private hospital/specialist rooms & $71(61)$ & $22(31)$ & $49(69)$ & \\
\hline \multicolumn{5}{|l|}{ Disease status } \\
\hline Resection completed - curative disease & $51(44)$ & $15(29)$ & $36(71)$ & \multirow[t]{6}{*}{0.991} \\
\hline Resection failed - locally advanced disease & $12(10)$ & $3(25)$ & $9(75)$ & \\
\hline Resection failed - metastatic disease & $3(3)$ & $1(33)$ & $2(65)$ & \\
\hline No resection - locally advanced disease & $14(12)$ & $4(29)$ & $10(71)$ & \\
\hline No resection - metastatic disease & $32(28)$ & $11(34)$ & $21(66)$ & \\
\hline No resection - because of age/comorbidities & $4(3)$ & $1(25)$ & $3(75)$ & \\
\hline \multicolumn{5}{|l|}{ Number of comorbidities } \\
\hline Mean (SD) & $2.2(1.4)$ & $2.5(1.6)$ & $2.0(1.4)$ & 0.100 \\
\hline \multicolumn{5}{|l|}{ Had chemotherapy } \\
\hline No & $17(15)$ & $5(29)$ & $12(71)$ & \multirow[t]{3}{*}{0.880} \\
\hline Yes & $96(83)$ & $30(31)$ & $66(69)$ & \\
\hline Not stated & $3(3)$ & - & - & \\
\hline \multicolumn{5}{|l|}{ Anxiety } \\
\hline Non-case & $67(58)$ & $28(42)$ & $39(58)$ & \multirow[t]{2}{*}{0.001} \\
\hline Sub-clinical/clinical case & $49(42)$ & $7(14)$ & $42(86)$ & \\
\hline \multicolumn{5}{|l|}{ Depression } \\
\hline Non-case & $61(53)$ & $27(44)$ & $34(56)$ & \multirow{2}{*}{0.001} \\
\hline Sub-clinical/clinical case & $55(47)$ & $8(15)$ & $47(85)$ & \\
\hline Social/family wellbeing & & & & \\
\hline Mean (SD) & $23.6(4.7)$ & $24.0(5.0)$ & $23.4(4.5)$ & 0.500 \\
\hline Pain & & & & \\
\hline Not at all/a little bit & $77(68)$ & $32(42)$ & $45(58)$ & $<0.001$ \\
\hline Somewhat/quite a bit/very much & $36(32)$ & $1(3)$ & $35(97)$ & \\
\hline Had a care coordinator/care plan & & & & \\
\hline No/not stated & $83(72)$ & $27(33)$ & $56(67)$ & 0.430 \\
\hline Yes & $32(28)$ & $8(25)$ & $24(75)$ & \\
\hline Accessed palliative care at baseline & & & & \\
\hline No & $65(56)$ & $19(29)$ & $46(71)$ & 0.800 \\
\hline Yes & $51(44)$ & $16(31)$ & $35(69)$ & \\
\hline
\end{tabular}

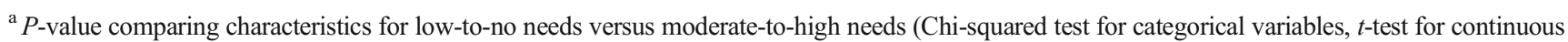
variables) 